\title{
Very high energy gamma-rays and the Hubble parameter
}

\author{
Alexia Gorecki, Aurélien Barrau* \\ Laboratoire de Physique Subatomique et de Cosmologie, CNRS/UJF/INPG \\ 53, rue des Martyrs 38026 Grenoble Cedex, France \\ *E-mail: barrau@lpsc.in2p3.fr \\ Julien Grain \\ Institut d'Astrophysique Spatiale, Université Paris-Sud 11, CNRS \\ Bâtiments 120-121, 91405 Orsay Cedex, France \\ Elisabetta Memola \\ Istituto Nazionale di Fisica Nucleare, INFN - Milano Bicocca \\ Piazza della Scienza 3, 20126 Milano, Italy
}

\begin{abstract}
A new method, based on the absorption of very high-energy gamma-rays by the cosmic infrared background, is proposed to constrain the value of the Hubble constant. As this value is both fundamental for cosmology and still not very well measured, it is worth developing such alternative methods. Our lower limit at the $68 \%$ confidence level is $H_{0}>$ $74 \mathrm{~km} / \mathrm{s} / \mathrm{Mpc}$, leading, when combined with the HST results, to $H_{0} \approx 76 \mathrm{~km} / \mathrm{s} / \mathrm{Mpc}$. Interestingly, this value, which is significantly higher than the usually considered one, is in exact agreement with other independent approaches based on baryonic acoustic oscillations and X-ray measurements. Forthcoming data from the experiments HESS-2 and CTA should help improving those results. Finally, we briefly mention a plausible correlation between absorption by the extragalactic background light and the absence of observation of gamma-ray bursts (GRBs) at very high energies.
\end{abstract}

As the very high energy photons emitted by Active Galactic Nuclei (AGN) travel, they interact with the Cosmic Infrared Background (CIB) photons by electronpositron pair production. The cross section is maximum for the highest energies (typically around $10-20 \mathrm{TeV}$ ) and the interaction takes place with CIB photons in the range $1-100 \mu \mathrm{m}$. Assuming a well defined CIB energy density, the observed AGN spectrum can be unfolded. The absorption factor entering the unfolding procedure depends on the Hubble parameter, therefore it can be easily constrained by comparing the unfolded spectrum with theoretically "allowed" spectra. Quite simply, the smaller the Hubble constant, the higher the distance to the source, the higher the absorption, the harder the unfolded spectrum. As a consequence, a too small value of the Hubble parameter can eventually lead to an unphysical unfolded spectrum.

The CIB is the relic emission of the formation and evolution of galaxies of all types and star-forming systems. The near-IR CIB, from 1 to $10 \mu \mathrm{m}$, arises mainly from the stellar component of galaxies (probing the evolution at early times). For higher wavelengths, between 10 and $200 \mu \mathrm{m}$, the radiation is mostly due to dusty Ultra Luminous InfraRed Galaxies. The measure of the CIB spectrum is challenging as it is difficult to distinguish between the foreground, the atmosphere, and the instrument emission itself. Therefore, different methods are used in order to determine the CIB all along the emission range. We have considered an up-to-date CIB 
measurement inventory ${ }^{1-9}$, which satisfactorily constrains the spectrum.

To turn down the unfolded spectrum into a lower limit on $H_{0}$, one has to assume a class of "possible" source emission spectra. It is well known that the InverseCompton (IC) upper energy range bump mimics the concave Synchrotron bump (observed at lower energy). Moreover, there is no physical process to inject energy above the IC bump, and the Klein-Nishina effect tends to soften the spectrum at high energy. As a consequence, the main hypothesis of this study is to assume that the AGN spectrum must be concave at high energy, which is in agreement with the approach of Ref. 10 and Ref. 11. The blazar Mrk 501, with redshift $z=0.034$, is a good source candidate because it has been intensively observed in a broad dynamical range between $400 \mathrm{GeV}$ and $21 \mathrm{TeV}$ during the 1997 flare by the experiments CAT and HEGRA, as reported in Ref. 12 and Ref. 11.

The unfolded spectrum is related with the observed spectrum by a multiplicative factor which is the exponential of the optical depth $\tau$. This optical depth depends on the cross section $\sigma\left(E_{A G N}, \epsilon_{C I B}\right)$ for $\gamma-\gamma$ pair production ${ }^{13}$ being $E$ and $\epsilon$ the observed energies at $z=0$, on the CIB energy density $n\left(\epsilon_{C I B}\right)$, and on the Hubble parameter as follows: $\tau \propto \frac{1}{H_{0}} \int n\left(\epsilon_{C I B}\right) \sigma\left(E_{A G N}, \epsilon_{C I B}\right) d \epsilon_{C I B}$. A Monte Carlo simulation was performed: at each step, for each wavelength, the CIB flux is randomly selected according to a Gaussian law centered at the best CIB estimate at the considered wavelength (the width is taken as the experimental error bar). This procedure is repeated for several $H_{0}$ values. Figure 1 shows the unfolded spectrum $\nu F_{\text {unfolded }}(\nu)=e^{\tau\left(H_{0}\right)} \nu F_{\text {obs }}(\nu)$, for different values of $H_{0}$. One can see that if the value of $H_{0}$ is too small, the unfolded spectrum is no longer concave, which is unphysical. Taking into account the uncertainties, the concavity requirement leads to $H_{0}>74 \mathrm{~km} / \mathrm{s} / \mathrm{Mpc}$ at the $68 \%$ confidence level (and to $H_{0}>54 \mathrm{~km} / \mathrm{s} / \mathrm{Mpc}$ at $90 \%$ confidence level) ${ }^{14}$.

Finally, it is worth mentioning that absorption by the extragalactic background light could very well play an important role in understanding the relatively rare population of GRBs observed above $10 \mathrm{GeV}$. So far, the highest energy emitted from a gamma-ray burst has been measured by the Fermi Large Area Telescope, that observed a photon $^{15}$ of $33.4_{-3.5}^{+2.7} \mathrm{GeV}$ from the GRB 090902B at $\mathrm{z}=1.822$. Although slightly smaller than Stecker values ${ }^{16}$, our raw estimates of the optical depth is of the same order $(\tau \sim 5)$. This would lead to huge absorption factors of the order of 150, making the unfolded spectrum quite unphysical. Evolution effects should of course be correctly taken into account, but the situation is quite puzzling. 


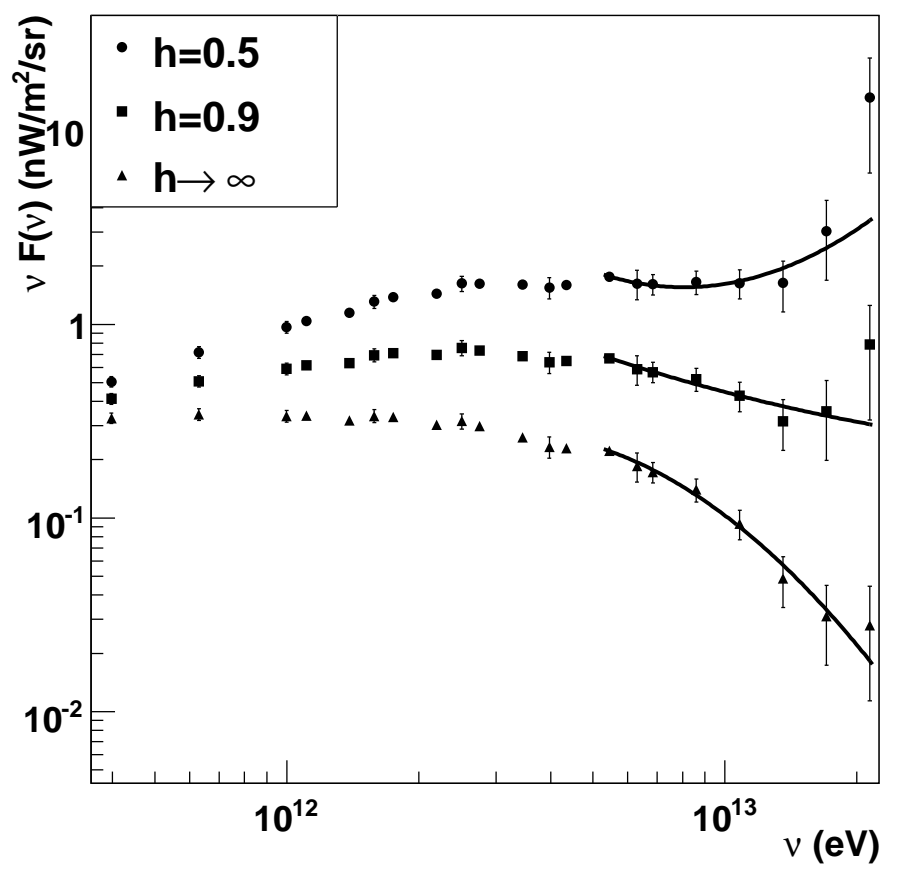

Fig. 1. Unfolded gamma-ray spectra and associated fits for (from bottom to top): no CIB (equivalent to $\left.H_{0} \rightarrow \infty\right), H_{0}=50 \mathrm{~km} / \mathrm{s} / \mathrm{Mpc}$, and $H_{0}=90 \mathrm{~km} / \mathrm{s} / \mathrm{Mpc}$.

\section{References}

1. Bernstein, R.A., Freedman, W.L., Madore, B.F., ApJ, 571, 107 (2002).

2. Cambresy, L., Reach, W.T., Beichman, C.A., Jarett, T.H., ApJ, 555, 563 (2001).

3. Dole, H., Lagache, G., Puget, J.-L., et al., A $\& A$, 451, 417 (2006).

4. Elbaz, D., Cesarsky, C.J., Fadda, D. et al., A\&A, 351, L37 (1999).

5. Gorjian, V., Wright, E.L., Chary, R.R., ApJ, 536, 550 (2000).

6. Matilla, K., ApJ, 591, 119 (2003).

7. Papovich, C., Dole, H., Egami, E. et al., ApJS, 154, 70 (2004).

8. Savage, R.S., Oliver, S., Statistical constraints on the IR galaxy number counts and cosmic IR background from the Spitzer GOODS survey (2005).

9. Wright, E.L., ApJ, 553, 748 (2001).

10. Renault, C., Barrau, A., Lagache, G., Puget, J.-L., A 6 A, 371, 771 (2001).

11. Guy, J., Renault, C., Aharonian, F., Rivoal, M., Tavernet, J.-P., A $E A$, 359, 419 (2000).

12. Aharonian, F.A., Akhperjanian, A.G., Barrio, J.A., Bernlöhr, K. et al., A $\& A$, 349, 11 (1999).

13. Heitler, W., The Quantum Theory of Radiation, (Oxford, 1960).

14. Barrau, A., Gorecki, A. and Grain, J., MNRAS, 389, 919 (2009).

15. Abdo et al., ApJL, 706, L138 (2009).

16. Stecker, F., Malkan, M.A., Scully, S.T., ApJ, 648, 774 (2006). 